\title{
Migraine Headaches: Feverfew or Chamomile Leaves?
}

\section{Snezana Agatonovic-Kustrin ${ }^{1 *}$, David Babazadeh Ortakand² and David W Morton ${ }^{2}$}

${ }^{1}$ Faculty of Pharmacy, Universiti Teknologi MARA (UiTM), Bandar Puncak Alam, Selangor 42300, Malaysia

${ }^{2}$ School of Pharmacy and Applied Science, La Trobe Institute of Molecular Sciences, La Trobe University, Bendigo 3550, Australia

\begin{abstract}
The purpose of this study was to compare and analyse active components in feverfew and chamomile using High Performance Thin Layer Chromatography as the analytical method. Both plants belong to the same Asteraceae family and feverfew is sometimes mistaken for German chamomile due to similar flowers. Feverfew leaves have been traditionally used in the treatment of migraine, with Parthenolide regarded as the primary active ingredient. On the other hand, bisabolol and chamazulene have anti-inflammatory properties, and are the main active components in German chamomile essential oil which is obtained by steam distillation of flower heads.
\end{abstract}

Bisabolol and chamazulene were present in higher concentrations in flowers and in leaves from flowering German chamomile. Parthenolide was present in higher concentration in leaves. Parthenolide and chamazulene are both terpenoids, derived from the same sesquiterpene precursor, farnesyl diphosphate, via two different biosynthetic pathways. Our study of Feverfew and German chamomile suggests that the Parthenolide pathway is favoured in leaves, while formation of matricin and bisabolol is favoured in flowers.

Anti-inflammatory activity of chamazulene and the presence of Parthenolide could explain and justify the use of chamomile in the treatment and prevention of migraine.

Keywords: Feverfew; German chamomile; HPTLC fingerprinting; Parthenolide; Bisabolol; Chamazulene

\section{Introduction}

Feverfew, also known as wild chamomile, has been traditionally used in the treatment of headache and migraine. Since clinical trials have confirmed its effectiveness against headaches and migraine [1]. Feverfew is recommended as a migraine prophylactic [2]. The most well-studied and abundant group of active compounds in feverfew are the sesquiterpene lactones produced by superficial leaf glands, with Parthenolide considered to be the main active ingredient $[3,4]$. Parthenolide can also be found in other Asteraceae species, such as German Chamomile [5].

Due to similar flowers, Feverfew (Chrysanthemum parthenium/ Tanacetum parthenium L.) is sometimes mistaken for German Chamomile (Matricaria Recutita) [6]. Although German chamomile belongs to the same Asteraceae family as Feverfew, its use in migraine prophylaxis is not evidenced. Pharmacological effect of German Chamomile is mainly associated with its essential oils extracted from the flowers. The main active constituents in essential oil are (-)- $\alpha$-bisabolol and its oxides, chamazulene [7], hydrophilic polyphenols, flavonoids (e.g., apigenin) [8] and their glucosides [9]. The essential oil of both German and Roman chamomile is a light blue in colour due to the presence of terpenoid chamazulene (5\%). Bisabolol comprises $50 \%$ of German chamomile's essential oil. Chamazulene and bisabolol are present in higher concentrations in German than in Roman chamomile. Since Parthenolide, alpha-bisabolol, matricin and chamazulene, all belong to sesquterpenes, a class of terpenes that consist of three isoprene units, the aim of this study was to compare High Performance Thin Layer Chromatographic (HPTLC) fingerprints of Feverfew and German chamomile and to quantify chamazulene, bisabolol and Parthenolide in fresh leaf and flower heads. Parthenolide content of dried leaf deteriorates on storage, and many commercial feverfew preparations have been shown to contain Parthenolide in concentrations well below the stated content [10] or even absent in some formulations [11].

Research comparing of Feverfew and German chamomile regarding its biologically active compound is lacking; furthermore evidence of Parthenolide content in German chamomile leaf extracts is limited. The current study aims to compare the levels of bisabolol, chamazulene and Parthenolide in Feverfew and German Camomile extracts, both from flower heads and leaves, and rationalize the use of German chamomile leaves in anti-migraine therapy.

\section{Materials and Methods}

\section{Chemicals}

German Chamomile, Feverfew and Calendula plants were purchased from the local nursery (Bendigo Wholefoods and Nursery, Bendigo, Australia) and grown in growth chambers for 5 months at a constant temperature of $21^{\circ} \mathrm{C}$ using a 12 hour day/ night cycle. Standardised supercritical carbon dioxide $\left(\mathrm{CO}_{2}\right)$ extract of Feverfew and German Chamomile were donated by FLAVEX Naturextrakte $\mathrm{GmbH}$ (Rehlingen, Germany), (97\%) Aldrich (Milawauki, USA), Parthenolide were purchased from from Sigma-Aldrich (Munich, Germany), chamazulene and bisabolol from Phytolab (Vestenbergsgreuth, Germany). Glacial acetic acid and concentrated sulphuric acid were obtained from (Merck, Darmstadt, Germany). Anisaldehyde was purchased from ACROS organics (New Jersey, USA). Separations were performed on 20 $\times 10 \mathrm{~cm}$ normal phase Silica gel 60 F254 HPTLC plates. All solvents and chemicals used were of analytical grade. Acetone and acetic acid were purchased from Merck (Kilsyth, Victoria, Australia), hexane from BDH (Poole, England) ethyl acetate from Sigma-Aldrich (Munich, Germany), and Milli-Q (Millipore) water was used.

\section{Standard solutions}

$0.1 \mathrm{mg} / \mathrm{mL}$ standard solutions of bisabolol chamazulene and

*Corresponding author: Snezana Agatonovic-Kustrin, Faculty of Pharmacy Universiti Teknologi MARA (UiTM), Bandar Puncak Alam, Selangor 42300, Malaysia, Tel: +603-3258-4610; E-mail: snezana@puncakalam.uitm.edu.my

Received October 16, 2015; Accepted November 03, 2015; Published November 06, 2015

Citation: Agatonovic-Kustrin S, Ortakand DB, Morton DW (2015) Migraine Headaches: Feverfew or Chamomile Leaves?. Mod Chem appl 3: 169. doi:10.4172/2329-6798.1000169

Copyright: ( 2015 Agatonovic-Kustrin S, et al. This is an open-access article distributed under the terms of the Creative Commons Attribution License, which permits unrestricted use, distribution, and reproduction in any medium, provided the original author and source are credited. 
Parthenolide were prepared using absolute ethanol (Thermo Fisher Sci., Scoresby, Australia) and stored at $4^{\circ} \mathrm{C}$ to prevent degradation.

\section{Extraction}

$10 \mathrm{~g}$ of fresh leaves were picked and frozen. Frozen leaves were macerated into a fine paste in a mortar and pestle, placed into $22 \times$ $80 \mathrm{~mm}$ cellulose extraction thimble (Whatman, Little Chalfont, UK), and refluxed in a Soxhlet apparatus for 12 hours using $100 \mathrm{~mL}$ of absolute ethanol. The resulting extracted solution was concentrated to approximately $10 \mathrm{~mL}$ using a rotary evaporator (Buchi Rotavapor Model R-200), transferred into a $25 \mathrm{~mL}$ volumetric flask and adjusted to volume using absolute ethanol. All solutions were stored at $4^{\circ} \mathrm{C}$ to minimise degradation.

\section{High performance thin layer chromatography}

HPTLC plates were pre-washed before use with a blank run of ethanol, dried and activated, by heating in an oven at $110^{\circ} \mathrm{C}$ for 15 minutes. Samples were sprayed as $8 \mathrm{~mm}$ wide bands using a $100 \mu \mathrm{L}$ HPTLC syringe (Hamilton, Bonaduz, GR, Switzerland) with a semiautomatic sample applicator (Linomat 5, Camag, Muttenz, Switzerland), $8 \mathrm{~mm}$ from the lower edge, with $10 \mathrm{~mm}$ distance from each side, and a distance of $4 \mathrm{~mm}$ between each tracks ( 15 tracks in total).

Anisaldehyde reagent for post-chromatographic derivatization: The anisaldehyde spray reagent solution was freshly prepared before use by combining anisaldehyde with a refrigerated solution of glacial acetic acid / concentrated sulphuric acid (Merck, Darmstadt, Germany) in the ratio of $0.5: 50: 1$.

HPTLC plate development and visualisation: Chromatographic plates were developed in an Automated Multiple Development Chamber (AMD 2, Camag, Muttenz, Switzerland) using hexane: ethyl acetate: acetic $(20: 10: 1)$ acid as the mobile phase. Images of the developed plates for both standards and analysed extracts, were recorded using a TLC-visualiser (Camag, Muttenz, Switzerland) equipped with a 12-bit Charged Couple Device (CCD) digital camera and winCATS software (Camag, Muttenz, Switzerland) under UV light at $366 \mathrm{~nm}$ and $256 \mathrm{~nm}$, and white light above and below the plate. Developed plates were photographed before and after derivatization by spraying with anisaldehyde solution.

WinCATS image capturing parameters were fixed to ensure high quality images and reproducibility between plates. Quantitative HPTLC analysis was performed using VideoScan Digital Image Evaluation software (Camag, 2003) and set to recognise fluorescent bands.

\section{Method validation}

Linearity of the method for quantification of bisabolol, chamazulene and Parthenolide was assessed by plotting chromatographic peak area versus applied amounts of standards in $\mu \mathrm{g}$ over a range of: $0.2-6.0 \mu \mathrm{g}$ for bisabolol; 0.1-6.0 $\mu \mathrm{g}$ for chamazulene; and 0.1-6.0 $\mu \mathrm{g}$ for Parthenolide.

Regression analysis was performed using the least-squared method. Specificity was assessed by the capacity of the optimised mobile phase to separate applied standards. Repeatability of the method (intra assay precision) was assessed by applying replicates at two different concentrations within the linearity range. Variance between replicates was expressed as a Relative Standard Deviation (\%RSD). The detection limit (LOD) was calculated by multiplying the standard deviation of multiple measurements $(n=5)$ by 3 and then dividing by the slope of the calibration curve [12]. The Limit of Quantification (LOQ) [12] was determined from multiple measurements $(n=3)$ of the chromatographic response of a single sample mixture using Equation 1.

$$
\mathrm{LOQ}=\frac{10 \times \mathrm{Sd}}{\text { Slope }}
$$

\section{Results and Discussion}

A simple HPTLC method was developed for simultaneous determination of bisabolol, chamazulene and Parthenolide using a mixture of hexane: ethyl acetate: acetic (20:10:1) acid as the mobile phase. Bands were characterised by their $R_{f}$ values and colour after derivatization by spraying with anisaldehyde solution. Bisabolol, chamazulene and Parthenolide were observed after derivatization as purple, brown and navy blue coloured bands under visible light (Figure 1) and $R_{f}$ values of $0.75 \pm$ $0.009,0.86 \pm 0.005$ and $0.67 \pm 0.005$ respectively.

Parthenolide is present in higher concentrations in leaves than in flower heads, while chamazulene and bisabolol are found in larger quantities in flowers. Furthermore, when compared Chamomile extracts, chamazule and bisabolol are found in leaves extracted from flowering German Chamomile plants while their content was bellow limit of detection in leaves obtained from non-flowering German and Roman chamomile plants (Figure 2). Parthenolide is found in leaves only and could not be detected in flower head extracts

Digitized images of plant extracts and standards from the plates were converted to chromatograms before analysis (Figure 3 ).

Linearity of the method was achieved in the concentration range of 0.5-4.0 $\mu \mathrm{g}$ for bisabolol; 0.6-5.0 $\mu \mathrm{g}$ for chamazulene, and 0.3-2.0 $\mu \mathrm{g}$ for Parthenolide (Figure 4). The correlation coefficients for each analytes were greater than 0.98 (Table 1 ). Since the slopes were not significantly different from unity, the method did not show proportional error. Small $y$-intercept values suggested unbiased method.

The sensitivity of the method was determined by calculating LOD and LOQ. The LOD values were $0.30,0.22$ and $0.10 \mu \mathrm{g}$, while the LOQ values were found to be $1.00,0.78$ and $0.36 \mu \mathrm{g}$ for bisabolol, chamazulene and Parthenolide respectively.

Good repeatability of the method was confirmed from the coefficient of variation of five replicates at two different concentrations of standards within their calibration ranges (Table 2) with averaged coefficients of variation of $4.1 \%, 3.0 \%$ and $3.2 \%$ for bisabolol, chamazulene and Parthenolide respectively. The relatively low values of \%RSD show that the method is precise and can be used to quantify bisabolol, chamazulene and Parthenolide in plant extracts.

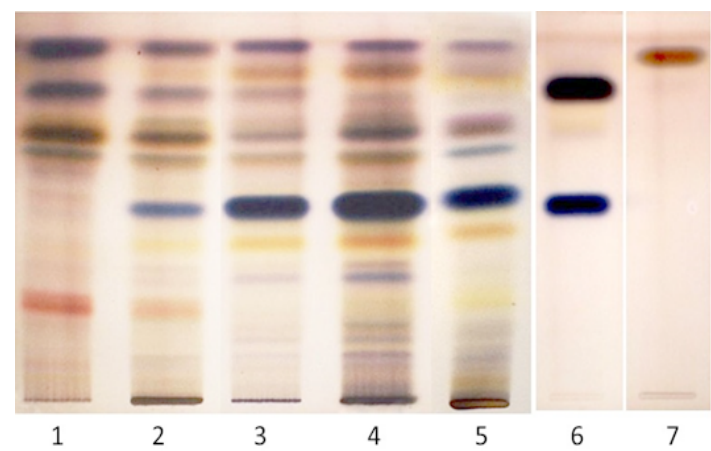

Figure 1: HPTLC fingerprints of chamomile and feverfew extracts and marker compounds. Mobile phase, hexane: ethyl acetate: acetic acid (20:10:1); derivatization with anisaldehyde reagent, white light under (tracks 1-6) and under $254 \mathrm{~nm}$ (track 7); from left to right: track 1, Chamomile supercritical flower head extract; track 2, Chamomile soxhlet leaf extract; track 3, Feverfew supercritical plant extract; track 4 Feverfew soxhlet leaf extract; track 5, Feverfew flower extract; track 6, parthenolide and bisablol; track 7, chamazulene; 


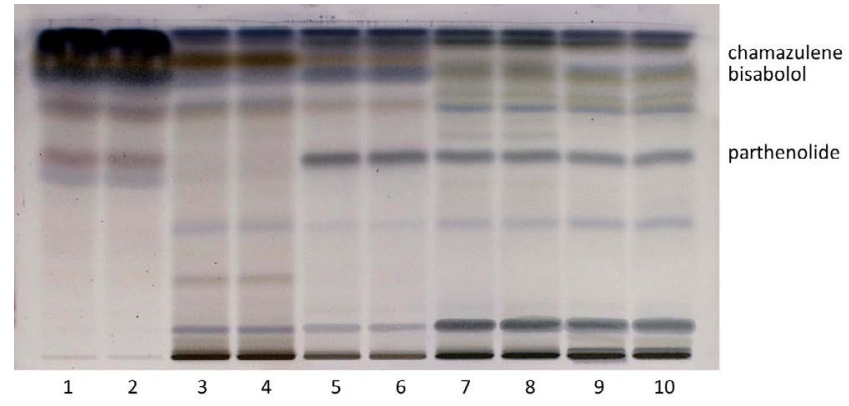

Figure 2: HPTLC fingerprints of chamomile extracts. Mobile phase, hexane: ethyl acetate: acetic acid (20:10:1); derivatization with anisaldehyde reagent, white light under; from left to right: tracks 1 and 2 , Chamomile flower heads; tracks 3 and 4 Chamomile supercritical flower head extract; track 5 and 6 , German Chamomile soxhlet leaf extract from flowering plant; track 7 and 8, Chamomile Roman Chamomile soxhlet leaf extract; tracks 9 and 10, german Chamomile soxhlet leaf extract.

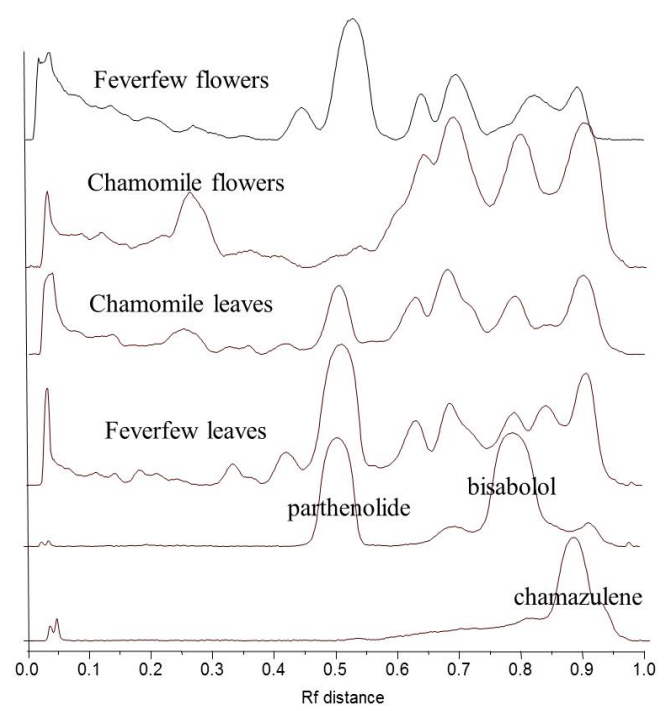

Figure 3: Superimposed chromatograms of feverfew and chamomile extracts with parthenolie, bisbolol and chamazulene chromatograms

The highest concentration of Parthenolide was found in Feverfew leaves $>$ Feverfew flowers $>$ Chamomile leaves $>$ Chamomile flowers. The highest amount of bisabolol was found in Chamomile flowers $>$ Chamomile leaves $>$ Feverfew flowers $\cong$ Feverfew leaves. The highest concentration of chamazulene was found in Chamomile flowers $>$ Chamomile leaves $>$ Feverfew flowers $>$ Feverfew flowers. Furthermore, Parthenolide cannot be quantified in Chamomile flowers.

The average bisabolol, chamazulene and Parthenolide concentrations in chamomile and feverfew extracts are given in Table 2.

Although the flowers are of great botanical importance, they are only a minor source of natural products that are used. Chamomile is among most important examples of plants whose flowers are used. The rational for use of Chamomile flowers in the treatment of headache and migraine can be explained with the antioxidant activity of bisabolol. It has been suggested that the formation of free radicals in the brain and the resultant oxidative damage may be involved in the pathogenesis of migraine [13] so it may be that the beneficial effects of Chamomile is due to the presence of free radical scavenging antioxidant compounds present in flowers.
Parthenolide and chamazulene are sesquiterpenes derived from the same sesquiterpene precursor, farnesyl diphosphate (FPP). The initial folding and cyclisation of FPP to sesquiterpenes is possible via six alternative reaction pathways [14], depending on the enzyme present in the tissue. Such tissue-specific synthesis of terpenoids is a widely reported phenomenon in a range of plant genera [15]. Different reactions lead to the formation of Parthenolide and matricin [16]. Our study suggests that the Parthenolide pathway is favoured in leaves, while formation of matricin (i.e., chamazulene) is favoured in flowers since higher concentrations of Parthenolide were found in leaves and higher concentrations of chamazulene were found in flowers.

Chamazulene is a thermal decomposition product from matricin, responsible for the dark blue coloration of the oil. Matricin becomes inflammatory through conversion to chamazulene [17]. Chamazulene is a natural profen, e.g., Nonsteroidal anti-inflammatory drug, an inhibitor of cyclooxygenase-2(COX-2), but not of cyclooxygenase-
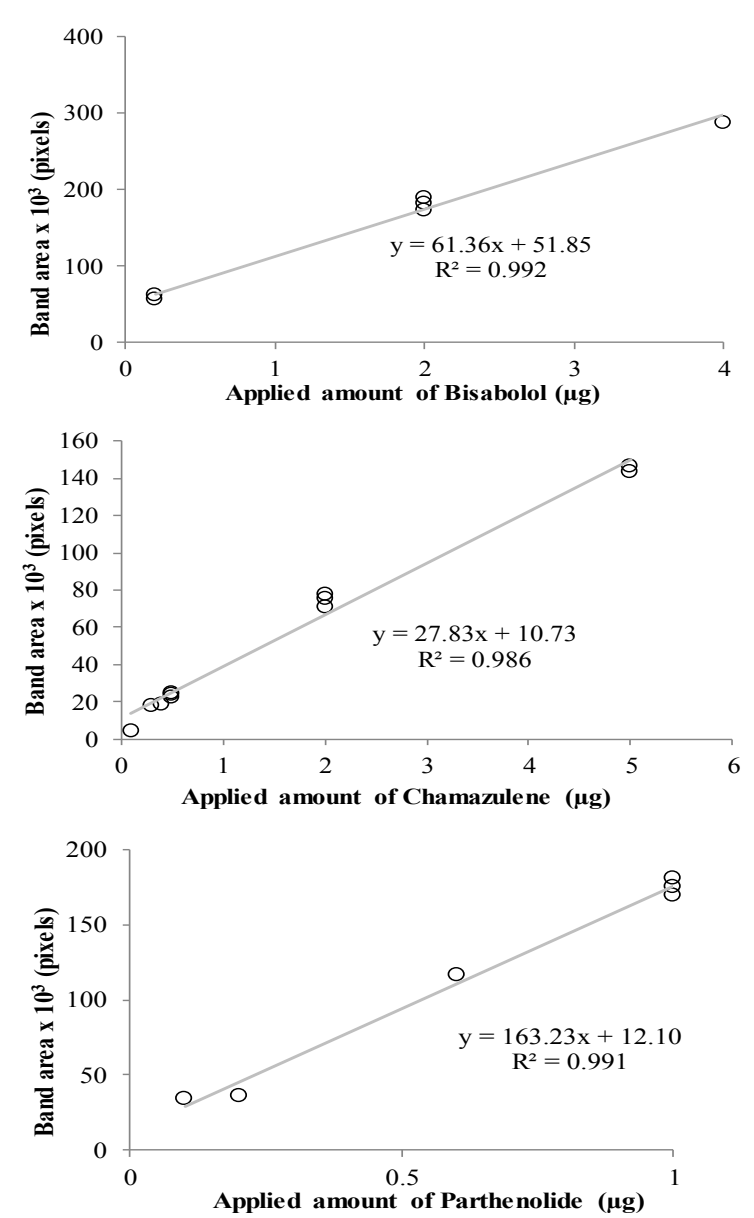

Figure 4: Calibration curves relating standard concentration to peak areas.

\begin{tabular}{|l|l|l|l|l|}
\hline Standard & Applied $(\boldsymbol{\mu g})$ & $\begin{array}{l}\text { Mean theoretical } \\
\text { value }(\boldsymbol{\mu g})\end{array}$ & $\begin{array}{l}\text { Mean recovery } \\
\mathbf{( \% )}\end{array}$ & RSD (\%) \\
\hline \multirow{2}{*}{ Bisabolol } & 2.00 & 2.09 & 104.39 & 5.30 \\
\hline \multirow{2}{*}{ Chamazulene } & 5.00 & 4.19 & 83.88 & 3.01 \\
\hline & 2.00 & 2.23 & 111.43 & 4.49 \\
\hline \multirow{2}{*}{ Parthenolide } & 5.00 & 4.68 & 93.65 & 1.51 \\
\hline & 1.11 & 1.10 & 109.67 & 5.35 \\
\hline
\end{tabular}

Table 1: Accuracy and precision of the method $(n=5)$. 
1(COX-1) [17]. Therefore matricin becomes inflammatory through conversion to chamazulene. Selective cyclooxygenase-2 inhibitors have been studied in acute migraine treatment based on the theory of upregulation of COX-2 in the inflammation involved in migraine pathophysiology [18].

The neurotransmitter serotonin (5-hydroxytryptamine [5HT]) plays a role in the development of migraine attacks [19]. This conclusion is supported by evidence indicating that migraine patients tend to have low levels of serotonin in their brains [20]. Although the exact mechanism that links abnormal 5-HT neurotransmission to the manifestation of head is not fully understood, a deficit on 5-HT descending pain inhibitory system is probably the most implicated in migraine pathophysiology. It has been suggested that Parthenolide may be a low-affinity antagonist at 5HT2A receptors [21]. Drugs that are serotonin receptor antagonists are used in migraine prevention (e.g., methysergide) [22]. In vitro studies have shown that Parthenolide, as well as other sesquiterpene lactones, inhibits serotonin release [10,23].

Assuming that molecules with similar geometry will have similar biological activities, we wanted to compare Parthenolide and chamazulene molecules with serotonin molecule. Molecular similarity is a pivotal concept in drug discovery and the cornerstone of StructureActivity Relationship (SAR) and structural clustering analysis. The rationale behind this approach is that structurally similar molecules are likely to have similar physicochemical properties [24], similar interactions with target receptors or enzymes (Active Analog Principle) [25] and should exhibit the same (or similar) biological activities.

The structure similarity can be calculated from the $2 \mathrm{D}$ structure fingerprint and expressed as Tanimoto coefficient [26] or from the 3D shape/pharmacophore superposition. The threshold of the shape similarity is $80 \%$, and of the feature (pharmacophore) similarity is $50 \%$. The most common 2D similarity calculation algorithms utilize molecular fingerprints as the similarity measure and capture the molecular connectivity/substructure/chemical feature information and analytical metrics are used to compare the implicit relationship between the two compounds, with the Tanimoto coefficient [26]. The 3D superposition algorithms are designed to find spatial similarity between molecules. 3D similarity measurements usually involve geometrical information of predefined objectives from the 3D molecular conformations, which include pharmacophores, molecular shapes, and molecular fields. During the 3D similarity calculation, 3D superposition is optimized by molecular shape/feature overlap and represented by the shape/ feature similarity score. Superposition of the molecules was done using on line PubChem 3D Viewer (https://pubchem.ncbi.nlm.nih.gov/ vw3d/vw3d.cgi) and similarity score was calculated using. The online PubChem services provide fast molecular similarity measure by $3 \mathrm{D}$ shape overlap and are expressed as similarity shape score. The targetindependent structure of the PubChem database, without any previous information on the physical or biological properties of the compounds, can be used to compare molecules [27]. Highly similar molecules may or may not have highly similar chemical or biological properties. Two molecules that are similar to a third molecule may be similar to each other or may not be similar.

Atoms of the one molecule are matched or assigned to the atoms of the other molecule and structures are superimposed. 3D alignment method requires consideration of the degrees of freedom that are related to the conformational flexibility of the molecules and is aimed to determine the alignment where similarity measure is at a maximum. The 3D superposition algorithms are designed to find spatial similarity between molecules following the paradigm that a necessary condition

\begin{tabular}{|l|l|l|l|l|l|}
\hline & \multicolumn{3}{|l|}{ Supercritical $\left.\mathbf{( C O}_{\mathbf{2}}\right)$} & \multicolumn{2}{l|}{ Soxhlet (Ethanol) } \\
\cline { 2 - 6 } & $\begin{array}{l}\text { Chamomile } \\
\text { flowers }\end{array}$ & $\begin{array}{l}\text { Feverfew } \\
\text { plant }\end{array}$ & $\begin{array}{l}\text { Chamomile } \\
\text { leaves }\end{array}$ & $\begin{array}{l}\text { Feverfew } \\
\text { flowers }\end{array}$ & $\begin{array}{l}\text { Feverfew } \\
\text { leaves }\end{array}$ \\
\hline Bisabolol & 2.15 & 1.30 & 0.53 & 0.47 & 0.42 \\
\hline Chamazulene & 5.35 & 3.35 & 1.71 & 0.66 & 1.58 \\
\hline Parthenolide & 0.49 & 0.59 & 0.38 & 0.33 & 0.75 \\
\hline
\end{tabular}

Table 2: Average concentrations $(n=3)$ of bisabolol, chamazulene and Parthenolide $(\mu \mathrm{g} / \mu \mathrm{L})$ found in Chamomile and Feverfew extracts.
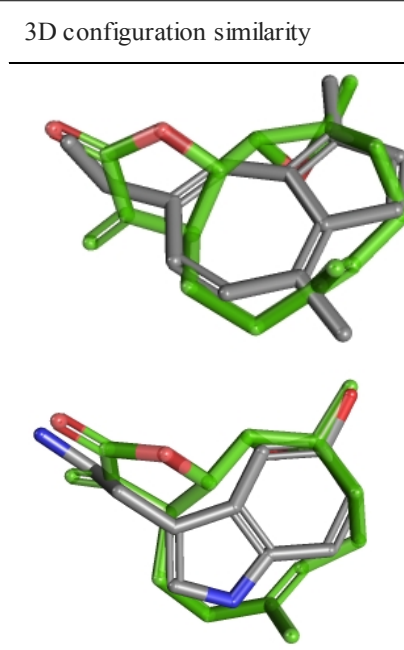

72

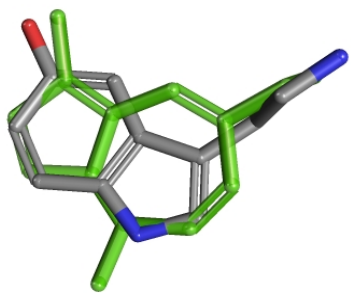

84

Figure 5: Superimposed a) chamazulene (gray) and parthenolide (green); b) parthenolide (green) and serotonin (gray) and c) chamazulene (green) and serotonin (gray).

for functional similarity is similar molecular geometry. To our surprise chamazulene has shown higher similarity (84\%) to serotonin than Parthenolide (72\%), while chamazulene and Parthenolide have $82 \%$ similarity score to each other (Figure 5). As a rule of the thumb, similarity greater than $80 \%$ provides $50 \%$ chance that the molecules will have similar activities [28].

\section{Conclusion}

Parthenolide is present in higher concentrations in leaves than in flower heads. Chamazulene and bisabolol are mainly found in flowers, with more observed in Chamomile flowers than in Feverfew flowers. Parthenolide and chamazulene are both terpenoids, both derived from the same sesquiterpene precursor farnesyl diphosphate via two possible biosynthetic pathways. Our study suggests that the Parthenolide pathway is favoured in leaves, while formation of matricin and bisabolol is favoured in flowers. Chamazulene is a natural profen (a non-steroidal anti-inflammatory drug). Selective cyclooxygenase-2 inhibitors have been studied in acute migraine treatment based on the theory of upregulation of COX-2 in the inflammation involved in migraine pathophysiology. The anti-inflammatory activity of chamazulene, and the presence of Parthenolide in both chamomile and feverfew, could explain and justify their use in the treatment and prevention of migraine. However, further research into the use of fresh German chamomile leaves in anti-migraine therapy is needed. 
Citation: Agatonovic-Kustrin S, Ortakand DB, Morton DW (2015) Migraine Headaches: Feverfew or Chamomile Leaves?. Mod Chem appl 3: 169. doi:10.4172/2329-6798.1000169

\section{References}

1. Vogler BK, Pittler MH, Ernst E (1998) Feverfew as a preventive treatment for migraine: a systematic review. Cephalalgia 18: 704-708.

2. Khan SI, Abourashed EA, Khan IA, Walker LA (2003) Transport of parthenolide across human intestinal cells (Caco-2). Planta Med 69: 1009-1012.

3. Piela-Smith TH, Liu X (2001) Feverfew extracts and the sesquiterpene lactone parthenolide inhibit intercellular adhesion molecule-1 expression in human synovial fibroblasts. Cell Immunol 209: 89-96.

4. Rios J, Passe MM (2004) Evidenced-based use of botanicals, minerals, and vitamins in the prophylactic treatment of migraines. J Am Acad Nurse Pract 16: $251-256$.

5. Chaves JS, Da Costa FB (2008) A proposal for the quality control of Tanacetum parthenium (feverfew) and its hydroalcoholic extract. Revista Brasileira de Farmacognosia 18: 360-366.

6. Smith RM, Burford MD (1993) Comparison of flavanoids in feverfew varieties and related species by principal components analysis. Chemometr Intell Lab Syst 18: 285-291.

7. Pino JA, Bayat F, Marbot R, Aguero J (2002) Essential oil of chamomile Chamomilla recutita (L.) Rausch. from Iran. J Essent Oil Res 14: 407-408.

8. Máday E, Szöke E, Muskáth Z, Lemberkovics E (1999) A study of the production of essential oils in chamomile hairy root cultures. Eur J Drug Metab Pharmacokinet 24: 303-308.

9. Mulinacci N, Romani A, Pinelli P, Vincieri FF, Prucher D (2000) Characterization of Matricaria recutita L. Flower extracts by HPLC-MS and HPLC-DAD analysis. Chromatographia 5: 301-307.

10. Marles RJ, Kaminski J, Arnason JT, Pazos-Sanou L, Heptinstall S, et al. (1992) A bioassay for inhibition of serotonin release from bovine platelets. J Nat Prod 55: 1044-1056.

11. Groenewegen WA, Heptinstall S (1986) Amounts of feverfew in commercial preparations of the herb. Lancet 1: 44-45.

12. Robinson KA (1986) Chemical Analysis. Little, Brown and Company, Boston.

13. Cordero MD, Cano-García FJ, Alcocer-Gómez E, De Miguel M, Sánchez-Alcázar JA (2012) Oxidative stress correlates with headache symptoms in fibromyalgia: coenzyme $Q_{10}$ effect on clinical improvement. PLoS One 7: e35677.

14. Dickschat JS (2011) Isoprenoids in three-dimensional space: the stereochemistry of terpene biosynthesis. Nat Prod Rep 28: 1917-1936.
15. Croteau R, Johnson MA (1984) Biosynthesis of terpenoids in glandular trichomes. In: Rodriguez E, Healey PL, Mehta L (Eds.), Biology and Chemistry of Plant Trichomes. Plenum Press, New York. pp: 133-185

16. Gutta P, Tantillo DJ (2006) Theoretical studies on farnesyl cation cyclization pathways to pentalenene. J Am Chem Soc 128: 6172-6179.

17. Ramadan M, Goeters S, Watzer B, Krause E, Lohmann K, et al. (2006) Chamazulene carboxylic acid and matricin: a natural profen and its natura prodrug, identified through similarity to synthetic drug substances. J Nat Prod 69: 1041-1045.

18. Loo CY, Tan HJ, Teh HS, Raymond AA (2007) Randomised, open label, controlled trial of celecoxib in the treatment of acute migraine. Singapore Med J 48: 834-839

19. Hamel E (2007) Serotonin and migraine: biology and clinical implications. Cephalalgia 27: 1293-1300.

20. Panconesi A (2008) Serotonin and migraine: a reconsideration of the centra theory. J Headache Pain 9: 267-276.

21. Weber JT, O'Connor MF, Hayataka K, Colson N, Medora R, et al. (1997) Activity of Parthenolide at 5HT2A receptors. J Nat Prod 60: 651-653.

22. Murphy JJ, Heptinstall S, Mitchell JR (1988) Randomised double-blind placebocontrolled trial of feverfew in migraine prevention. Lancet 2: 189-192.

23. De Weerdt CJ, Bootsma HP, Hendriks H (1996) Herbal medicines in migraine prevention Randomized double-blind placebo-controlled crossover trial of a feverfew preparation. Phytomedicine 3: 225-230.

24. Raevsky OA, Trepalin SV, Trepalina HP, Gerasimenko VA, Raevskaja OE (2002) SLIPPER-2001 -- software for predicting molecular properties on the basis of physicochemical descriptors and structural similarity. J Chem Inf Comput Sci 42: 540-549.

25. Maggiora GA, Johnson MA (1990) Concepts and applications of molecular similarity. American Chemical Society. Meeting. John Wiley, New York.

26. Baldi $P$, Nasr R (2010) When is chemical similarity significant? The statistical distribution of chemical similarity scores and its extreme values. J Chem Inf Model 50: 1205-1222.

27. Cincilla G, Thormann M, Pons M (2010) Structuring Chemical Space: SimilarityBased Characterization of the PubChem Database. Molecular Informatics 29: 37-49.

28. Taketo O, Akira K (2011) Metabolomics. Handbook of Molecular and Cellular Methods in Biology and Medicine, Third Edition. CRC Press. pp: 471-484. 